\title{
Can Self-Replicating Species Flourish in the Interior of a Star?
}

\author{
Luis A. Anchordoqui and Eugene M. Chudnovsky \\ Physics Department, Herbert H. Lehman College and Graduate School, The City University of New York \\ 250 Bedford Park Boulevard West, Bronx, NY 10468-1589, USA
}

\begin{abstract}
The existing view of biological life is that it evolves under suitable conditions in the low-temperature world of atoms and molecules on the surface of a planet. It is believed that any plausible extraterrestrial form of life must resemble the life on Earth that is ruled by the biochemistry of nucleic acids, proteins, and sugars. Going against this dogma, we argue that an advanced form of life based upon short-lived species can exist inside main-sequence stars like our Sun.
\end{abstract}

Keywords: nuclear life, cosmic necklaces

DOI: $10.31526 /$ LHEP.2020.166

Conventional wisdom states that the search for exolife calls for carbon-based organisms, operating in a water-based medium, with higher forms (perhaps) metabolizing oxygen. All forms of life on Earth share this same basic biochemistry. However, biological molecules are not stable in a thermodynamic sense [1]. Life can be thought out as a dynamical hypercycle (DNA encodes proteins, proteins help DNA to selfreplicate, DNA encodes proteins, etc.) that can survive indefinitely as long as free energy is available for it, and as long as self-replication goes faster than the destruction of information carriers. Mutations in the informational chain provide natural selection and evolution toward more complex species. Proteins are different chains of $20(+2)$ amino acids. To encode amino acids, one needs a minimum of a triplet RNA/DNA code of 4 nucleotides: A,C,G,U, like AAA, AUG, etc. This gives $4^{3}=64$ different combinations.

Of course, all this can be totally different for a different form of life, but self-replication is a cornerstone of life and it is hard to imagine how any life can be formed without encoding information. If one accepts that life is merely self-replication with mutations that leads to the increasing complexity through natural selection, any system capable of such processes can be viewed as a form of life. As an example, self-replicating robots assembling themselves in the environment that can be vastly different from the ambient conditions we are used to have long been considered one such possibility. More concretely, life needs at the minimum these three hypotheses:

1. The ability to encode information.

2. The ability of information carriers to self-replicate faster than they disintegrate.

3. The presence of free energy: at the minimum, $\Delta F=T \Delta S$, needed to constantly create order out of the disorder by decreasing entropy $S$ through self-replication, where $T$ is the temperature of the system.

Armed with this extreme reasoning, we ask whether some form of life could flourish deep inside the core of main-sequence stars like our Sun. We speculate that it is possible to satisfy hypotheses 1 and 3. Given how complex and nonobvious is the functioning of the biological cell that we are familiar with, and the fact that the early path of the biological evolution that has led to it is unknown, we will not speculate about the intricate series of bionuclear reactions that can satisfy hypothesis 2 . The only principle one should be guided with is that a self-replication hypercycle, if it exists, inevitably emerges in the dynamics of a complex system as a stationary process that survives out of all other processes.

It has long been suspected that any early universe phase transition occurring after inflation (say with symmetry breaking temperature $T_{c}$ ) may leave topological defects behind [2]. This is because when the system cools down below $T_{\mathcal{c}}$, there is some order parameter field multiplet $\phi$, which picks up a nonzero value $\phi_{0}$. If this were the case and the system has a symmetry group $G$, in the classical viewpoint, any operation $g \in G$ would transform the state $\phi$ into $g \phi_{0}$ [3]. Now, if we identify the subgroup leaving $\phi_{0}$ invariant with $H=\{h \in$ $\left.G \mid h \phi_{0}=\phi\right\} \subset G$, the manifold would be given by the quotient $\mathscr{M}=G / H$, namely, the set of left cosets $\{g H\}$ of $H$ in $G$. The topology of $\mathscr{M}$ characterizes the types of defects. For example, cosmic strings would form if the first homotopy group $\pi_{1}(\mathscr{M}) \neq 1$, where 1 stands for the group comprising the identity alone. Note that this implies that $\mathscr{M}$ contains closed loops that cannot be continuously shrunk to a point, and so, $\mathscr{M}$ would not be simply connected. Likewise, monopoles would be produced if $\pi_{2}(\mathscr{M}) \neq 1$. Note that for this particular configuration there would be nonshrinkable two-dimensional surfaces in $\mathscr{M}$.

Appealing to our discussion is the formation of composite defects. This may take place if a theory with symmetry group $G$ undergoes a phase transition (at $T_{\mathcal{C}_{1}}$ ) in which $\phi$ acquires a vacuum expectation value breaking the theory to a subgroup $H \subset G$, and after that, there is a second phase transition (at $T_{\mathcal{C}_{2}}$ ) in which the field $\psi$ gets a nonzero (but generally smaller) vacuum expectation value to break the symmetry further into $K \subset H$. One simple example is provided by the $S U(2)$ symmetry group. In the first stage of symmetry breaking, in which $\phi$ becomes nonzero the symmetry breaks down to $H=U(1)$ leading to the formation of 't Hooft-Polyakov monopoles [4, 5]. In a second stage, the symmetry breaks further into $K=\mathbb{Z}_{2}$ where strings would form. Remarkably, there is a stable configuration, the bead, in which the magnetic flux of the monopole $4 \pi / e$ is confined to two stable $\mathbb{Z}_{2}$ strings, each carrying a flux of $2 \pi / e[6,7]$. Such string structures with monopole beads are so-called "necklaces." Remarkably, multiple strings can originate from monopoles and connect them into two- and threedimensional structures, resembling atoms coupled by chemical bonds [8]. Since information needs to be written on a onedimensional chain to ensure easy reading and transcription [9], monopoles and antimonopoles of one kind would not be sufficient for that purpose. Each string carrying half of the magnetic flux that originates on a monopole has to go into antimonopole, thus making monopoles and antimonopoles of one kind alter- 

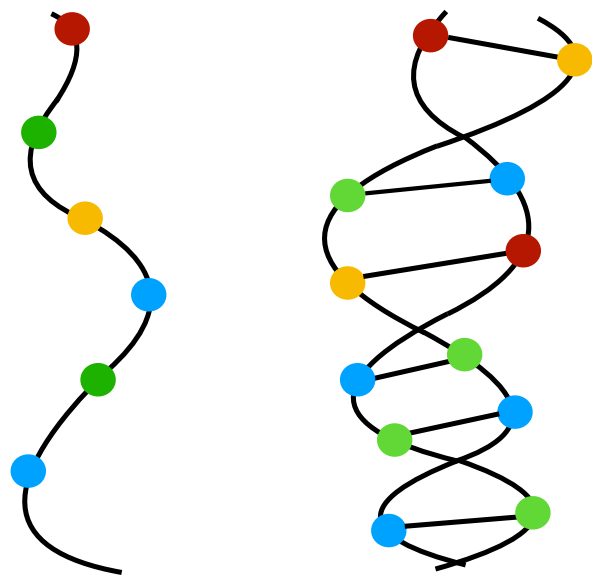

FIGURE 1: Encoding information with cosmic necklaces consisting of semipoles on strings (left), and a string double helix resembling DNA (right) [13].

nate in a one-dimensional chain. Such a chain would not carry any information. However, with various kinds of monopoles and non-Abelian cosmic strings discussed in the literature, it is easy to envision more complex sequences capable of encoding information. For example, two adjacent semipoles [10], unlike monopoles, need not have total charge zero and can repel each other instead of moving toward each other and annihilating [11, 12]. Such single- and double-chain structures formed by beads on necklaces (with a complex flux) are shown schematically in Figure 1. Semipoles correspond to an element of $\mathbb{Z}_{4}$ [10]. A semipole flux of $4 \pi\{1, i,-1,-i\} / e$ can be inferred from the differences between the fluxes of adjacent strings shown in Figure 1 , with $1 \equiv$ orange, $i \equiv$ green, $-1 \equiv$ red, and $-i \equiv$ blue. The flux configuration exhibited in Figure 1 reads $-1+i \leftrightharpoons 1+i \leftrightharpoons 1-i \leftrightharpoons-1-i \leftrightharpoons-1+i \leftrightharpoons-1-i \leftrightharpoons-1+i$.

The lifetime of such objects can be very short as far as their individual dynamics in a vacuum is concerned. It can be longer in a plasma of a star (see below) and can also be controlled by the metabolic process they encode. Note in this connection that any biological organism, when considered individually, is unstable. Compared to the lifetime of a star, its lifetime is an instantaneous spark of light in the dark. What is important is that such a spark manages to produce more sparks before it fades away, thus providing a long lifespan of the species. The complexity evolving through mutations and natural selection increases with the number of generations passed. Consequently, if lifetimes of self-replicating nuclear species are as short as lifetimes of many unstable composite nuclear objects are, they can quickly evolve toward enormous complexity.

We now turn to speculate on the capture of necklaces by stars and discuss hypothesis 3 . To develop some sense for the orders of magnitude involved, we assume that the strings are produced at the electroweak phase transition and so $T_{\mathcal{C}_{2}} \sim$ $100 \mathrm{GeV}$, yielding a mass per unit length of string $\mu \sim \hbar c^{3} T_{c_{2}}^{2} \simeq$ $10^{-6} \mathrm{~g} / \mathrm{cm}$. Before proceeding, we pause to note that for ultrarelativistic particles in thermal equilibrium, the blackbody formula gives the energy density-temperature relation $\varepsilon=C T^{4}$, where the constant $C$ depends on the number of degrees of freedom. For the electroweak phase transition, the energy density that must be deposited in the collision region to achieve the conditions for a phase transition, $\varepsilon \sim 10^{10} \mathrm{GeV} / \mathrm{fm}^{3}$, is 10 orders of magnitude larger than what is currently achieved in collisions at the CERN Large Hadron Collider [14].

In the spirit of [15], we consider superconducting strings (carrying electric currents and generating magnetic fields) that become magnetohydrodynamically frozen into plasma. The evolution process could begin with a single string in the system. The turbulence of the plasma stretches and twists the string loop, cutting it at the intersections, until an equilibrium network of string loops develops in which the process of stretching is balanced by the collapse of string loops due to tension. Note that the likelihood of a string being captured by a star is quite small. This is because the plasma wind from the star would prevent the string to approach it. A more probable scenario would be to assume that the string is captured during the star formation. The process of star formation begins in weakly ionized clouds of density in the range $10^{-22} \lesssim$ $\rho /\left(\mathrm{g} / \mathrm{cm}^{3}\right) \lesssim 10^{-20}$ [16]. Stars are formed as a result of slow gravitational condensation of matter in the high-density regions of the cloud. In the early stage of this process, neutral matter contracts together with plasma, and with the magnetic field and strings frozen into it. As the density increases, the degree of ionization falls and the neutral matter decouples from the plasma. During this stage, strings can be left out from the protostar. However, in the course of further contraction, the protostar becomes thermally ionized and recaptures its coupling to the magnetic field and to the strings. The radius of the ionized material is typically $R_{\text {ion }}=10^{8} \mathrm{~km}$. For typical clouds, the dispersion velocity of the turbulent motion is in the range $10^{5} \lesssim v /(\mathrm{cm} / \mathrm{s}) \lesssim 10^{6}[16]$. The force of friction per unit length of string produced by a superconducting string moving in a magnetized plasma is driven by the string velocity, the external magnetic field, and the plasma density. An order of magnitude estimate of the characteristic string length, $R_{*}$, can be obtained by balancing the frictional force per unit length with the force per unit length due to the tension in the string. If the motion of the turbulent plasma is well approximated by a Kolmogorov spectrum, $R_{*}$ is found to be

$$
R_{*} \sim 10^{9} \times \mu_{-6}^{1 / 3} \times v_{6}^{-2 / 3} \times \rho_{-20}^{-1 / 2} \mathrm{~km},
$$

where $\mu_{-6}$ is $\mu$ in units of $10^{-6} \mathrm{~g} / \mathrm{cm}, v_{6}$ is $v$ in units of $10^{6} \mathrm{~cm} / \mathrm{s}$, and $\rho_{-20}$ is $\rho$ in units of $10^{-20} \mathrm{~g} / \mathrm{cm}^{3}$ [15]. A simple estimate of the fraction of strings captured by the ionized plasma of the protostar is given by $\left\langle\left(R_{\text {ion }} / R_{*}\right)^{2}\right\rangle$. A straightforward calculation shows that a significant fraction of stars could be contaminated with strings.

Cosmic strings are classical solutions of relativistic fieldtheory models that are analogous to flux lines in superconductors. Such analogy arises from the mathematical equivalence of, e.g., Abelian Higgs model and Landau-Ginzburg theory of superconductivity. We will use this analogy to speculate on how the strings captured by the star could create a network of necklaces. There are processes in condensed matter in which a string loop breaks into a string with open ends capped by monopole and antimonopole (which corresponds to the formation of the monopole-antimonopole pair on a string); see, e.g., $[17,18,19,20]$. This analogy could also be translated to hadron physics, where the quarks would act as the carriers of magnetic charge permanently bound in pairs by the string bonds [21]. If besides stretching, interconnecting, and forming new loops, the turbulent plasma also breaks them into segments capped by monopoles, the turbulence would produce 


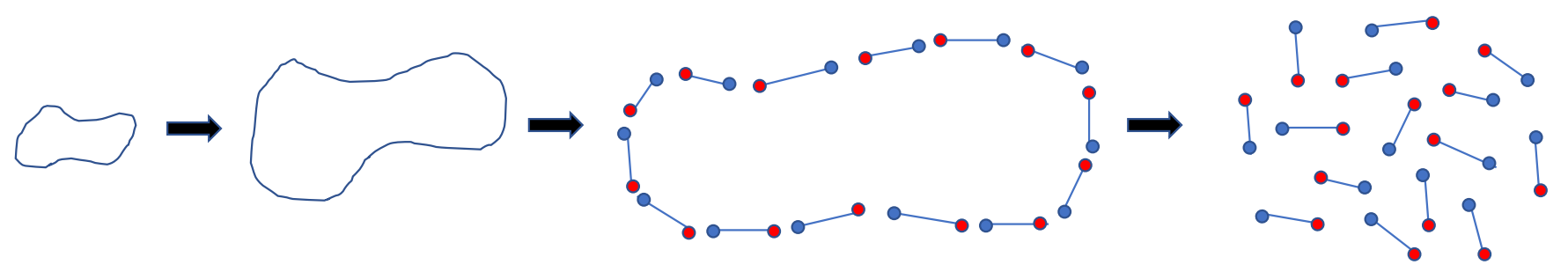

FIGURE 2: Turbulence, besides stretching loops, can cut them into segments bounded by monopole-antimonopole pairs.

monopoles together with networks of strings; see Figure 2. Small segments of strings capped by monopoles formed in this way can be the building blocks of longer information carriers once they split into semipoles to form cosmic necklaces. Information stored in a cosmic necklace must encode nuclear species that, by analogy with the DNA-protein machinery, must assist replication of necklaces. The details of such process may be as complicated as the details of the primordial self-replication that led to the origin of the biological cell, which are unknown. Life on Earth is the only proof that such mechanism exists. For that reason, we will not go beyond the ability of nuclear species to store information.

Consumption of free energy by biological life on Earth has been constantly growing with time, starting with the use of solar energy, then utilizing in the last 200 years even greater amounts of solar energy accumulated in fossil fuels, and then proliferating to the consumption of nuclear power thanks to complexity developed from that massive most recent consumption of energy. By analogy, nuclear life, if it develops, must accelerate burning of nuclear fueled by stars. Indeed, life in the interior of a star would change its thermodynamics as a fraction of the fusion energy would be used for self-replication, and for developing and maintaining a sophisticated order. Consequently, one way to search for nuclear life in the universe would be to look for stars whose behavior defies explanation based upon conventional theories.

Consider, for example, the flow of energy from the hot surface of a star into the open space. When the temperature gradient in the surface layer reaches a critical value, the transfer of heat from the hot bottom of the layer to the colder top switches from heat conduction to convection via formation of Benard cells, which is a trivial textbook example of self-organization. Hot plasma rises up from the bottom of the layer through the center of each cell, cools down at the top of the layer, and then sinks inside the walls of the cell. This accelerates the transfer of heat. The general principle behind it is the formation of structures that allow the fastest release of the free energy possessed by the star. The surface cools faster due to such selforganization.

In a similar way, from a thermodynamic point of view, the formation of the nuclear life in the interior of a star will have a "purpose" to provide faster escape of the energy released by nuclear fusion from the core of the star toward its surface. It makes the star to cool faster via a complex process of selforganization maintained by the elevated flux of energy through it. This would provide the same end effect as advocated in [22] to explain the observed deviations from predictions of models of stellar evolution, which seem to indicate an over-efficient stellar cooling.
We will conclude by mentioning one such example. The NASA's Kepler and K2 missions provided long-baseline and high precision photometry for hunting exoplanets by searching for regular decreases in the emission light of a star caused by a planet passing between the star and the telescope on its orbit. These passes are called transits. Very recently, the K2 Team reported that EPIC 249706694 (HD 139139), which is about 360 light years away, experienced 28 transient-like events over the course of its 87-day observation period [23]. The depths in the flux during these events are roughly $200 \pm 80$ parts per million, with durations varying between 1 and 7 hours and mean separation time of about 3 days. These intriguing dips, which would normally indicate an orbiting system of planets, exhibit no obvious periodicity, and moreover, the K2 Team concluded that their arrival time could have been produced by a random number generator. The target object is a relatively unevolved star of spectral type $G$, with solar effective temperature and radius. The star rotation period of about 14.5 days yields a gyrochronological age of $1.5 \pm 0.4$ Gyr. However, in the same photometric aperture, there is a fainter neighboring star, somewhat cooler $(4400 \mathrm{~K})$, and it is separated from the target star by $3.3^{\prime \prime}$. There is insufficient Gaia kinematic data for the neighboring star to determine whether it is bound or not to the target star. Because of this, there is no certainty which star actually hosts the transit-like events. The K2 Team estimated that if the dips are real transits across the target star, then the inferred planet size would be approximately 2 Earth's radii, whereas if they shadow the neighboring star, then the planetary radii would be more like 1-2 times Jupiter's size. All of the simple explanations have been ruled out by the K2 Team.

Of course, attributing the anomaly in the luminosity of a star to nuclear life would be a very long shot. We just wanted to emphasize that such unexplained anomalies do exist. Maybe, the answer to Fermi's question: Where is everybody? can be found in the least expected places.

\section{ACKNOWLEDGMENTS}

We are grateful to Alex Vilenkin and Kari Rummukainen for useful comments. This work has been supported by the U.S. National Science Foundation (NSF Grant PHY-1620661) (L.A.A.), the National Aeronautics and Space Administration (NASA Grant 80NSSC18K0464) (L.A.A.), and the U.S. Department of Energy (DOE Grant DE- FG02-93ER45487) (E.M.C). Any opinions, findings, and conclusions or recommendations expressed in this material are those of the authors and do not necessarily reflect the views of the NSF, NASA, or DOE. 


\section{References}

[1] E. M. Chudnovsky, Thermodynamics of natural selection, J. Statist. Phys. 41, 877 (1985) doi:10.1007/BF01010008

[2] T. W. B. Kibble, Some implications of a cosmological phase transition, Phys. Rept. 67, 183 (1980). doi:10.1016/03701573(80)90091-5

[3] T. W. B. Kibble, Symmetry breaking and defects, [arXiv:condmat/0211110 [cond-mat]].

[4] G. 't Hooft, Magnetic monopoles in unified gauge theories, Nucl. Phys. B 79, 276 (1974). doi:10.1016/05503213(74)90486-6

[5] A. M. Polyakov, Particle spectrum in the quantum field theory, JETP Lett. 20, 194 (1974) [Pisma Zh. Eksp. Teor. Fiz. 20, 430 (1974)].

[6] M. Hindmarsh and T. W. B. Kibble, Monopoles on strings, Phys. Rev. Lett. 55, 2398 (1985). doi:10.1103/PhysRevLett.55.2398

[7] T. Vachaspati and A. Vilenkin, Evolution of cosmic strings, Phys. Rev. D 35, 1131 (1987). doi:10.1103/PhysRevD.35.1131

[8] T. W. B. Kibble and T. Vachaspati, Monopoles on strings, J. Phys. G 42, no. 9, 094002 (2015) doi:10.1088/09543899/42/9/094002 [arXiv:1506.02022].

[9] E. P. Wigner, The probability of the existence of a selfreproducing unit, in The Logic of Personal Knowledge: Essays Presented to Michael Polanyi on his Seventieth Birthday, p. 231. Routledge \& Kegan Paul, London, 1961.

[10] M. Hindmarsh, K. Rummukainen and D. J. Weir, New solutions for non-Abelian cosmic strings, Phys. Rev. Lett. 117, no.25, 251601 (2016) doi:10.1103/PhysRevLett.117.251601 [arXiv:1607.00764 [hep-th]].

[11] M. Hindmarsh, K. Rummukainen and D. J. Weir, Numerical simulations of necklaces in SU(2) gauge-Higgs field theory, Phys. Rev. D 95, no.6, 063520 (2017) doi:10.1103/PhysRevD.95.063520 [arXiv:1611.08456 [astro-ph.CO]].

[12] M. Hindmarsh, A. Kormu, A. Lopez-Eiguren and D. J. Weir, Scaling in necklaces of monopoles and semipoles, Phys. Rev. D 98, no.10, 103533 (2018) doi:10.1103/PhysRevD.98.103533 [arXiv:1809.03384].

[13] It is believed that microbial life first developed with RNA and then evolved into cells having DNA as the volume of heredity material increased. Damage in the DNA is easily repaired due to pairing in the double chain, which is basically the error correction mechanism. It requires one chain to be the mirror image of the other. Various kinds of beads discussed in literature can, in principle, provide such mirror pairing in a necklace double helix but we leave it to others to speculate about specific realizations.

[14] D. Boyanovsky, H. J. de Vega and D. J. Schwarz, Phase transitions in the early and the present universe, Ann. Rev. Nucl. Part. Sci. 56, 441 (2006) doi:10.1146/annurev.nucl.56.080805.140539 [hepph/0602002].

[15] E. Chudnovsky and A. Vilenkin, Strings in the Sun?, Phys. Rev. Lett. 61, 1043 (1988). doi:10.1103/PhysRevLett.61.1043

[16] M. R. Krumholz, The big problems in star formation: the star formation rate, stellar clustering, and the initial mass function, Phys. Rept. 539, 49 (2014) doi:10.1016/j.physrep.2014.02.001 [arXiv:1402.0867].
[17] M. C. Diamantini, C. A. Trugenberger and V. M. Vinokur, Quantum magnetic monopole condensate, [arXiv:2007.02356 [hep-th]].

[18] L. D. C. Jaubert and P. C. W. Holdsworth, Signature of magnetic monopole and Dirac string dynamics in spin ice, Nature Phys. 5, 258-261 (2009) doi:10.1038/nphys1227 [arXiv:0903.1074 [cond-mat.stat-mech]].

[19] E. Mengotti, L. J. Heyderman, A. Fraile Rodriguez, F. Nolting, R. V. Hugli and H. B. Braun, Real-space observation of emergent magnetic monopoles and associated Dirac strings in artificial kagome spin ice, Nature Phys. 7, 68-74 (2011) doi:10.1038/nphys1794

[20] D. A. Garanin, E. M. Chudnovsky, and T. Proctor, The role of vortices in the three-dimensional random-field $x y$ model EPL 103, 67009 (2013) doi:10.1209/0295-5075/103/67009

[21] Y. Nambu, Strings, monopoles and gauge fields, Phys. Rev. D 10, 4262 (1974) doi:10.1103/PhysRevD.10.4262

[22] M. Giannotti, I. Irastorza, J. Redondo and A. Ringwald, Cool WISPs for stellar cooling excesses, JCAP 05, 057 (2016) doi:10.1088/1475-7516/2016/05/057 [arXiv:1512.08108 [astro-ph.HE]].

[23] S. Rappaport et al. The random transiter - EPIC 249706694/ HD 139139, Mon. Not. Roy. Astron. Soc. 488, 2455 (2019) doi:10.1093/mnras/stz1772 [arXiv:1906.11268]. 